\title{
Analysis on magnetic flux density and core loss for hexagonal and butt-lap core joint transformers
}

\begin{abstract}
This paper presents the results of new hexagonal configuration at the T-joint of three-phase transformer core. The proposed model is compared with previous T-joint design, Butt-lap, which is widely used at present by many transformer manufacturers. The magnetic flux density distribution and core loss of a transformer rated 1000kVA are analyzed for the two types of T-joint design. The 3D simulation are carried out by using the ANSYS-Maxwell software. The results show that the magnetic flux density of the hexagonal shape T-joint is well distributed compared to that in the Butt-lap T-joint design. The core loss for the proposed model (hexagon) T-joint indicates a reduction of more than $11 \%$ compared to the Butt-lap T-joint design.
\end{abstract}

Keyword: Core losses; Joints design; Magnetic flux density; Power transformer 